\title{
Implementing a Testbed for Mobile Multimedia
}

\author{
A.Dutta, J.C. Chen, S. Das, M. Elaoud, D. Famolari, S.Madhani, A. McAuley, M. Tauil \\ Telcordia Technologies Inc., 445 South Street, Morristown, NJ 07960 \\ S. Baba, T. Maeda, N. Nakajima, Y. Ohba \\ Toshiba America Research Inc., Morristown, NJ 07960 \\ Henning Schulzrinne \\ Computer Science Department, Columbia University, New York, NY 10027
}

\begin{abstract}
-
In an effort to realize wireless Internet telephony and multimedia streaming in a highly mobile environment a testbed emulating a wireless Internet has been built. This would allow setting up multi-media calls between IP mobiles and integration between IP and PSTN end-points in a wireless environment. Different functionalities and components involved with the wireless Internet streaming multimedia have been prototyped and experimented in the testbed. These include signaling, registration, dynamic binding, location mangamenet as well as supporting the QoS features for the mobile users. This paper describes some of the components of the testbed and highlights the experiences while building this testbed which could be beneficial to some who plan to build a similar testbed to realize several features and capabilities of Mobile Wireless Internet, before actually bringing to the market.
\end{abstract}

\section{INTRODUCTION}

Providing flexible and programmable multimedia services at a lower cost to the end users in a more efficient way has been one of the main motivation behind transitioning to a packet based network from a traditional circuit switched network. Most of the efforts underway now are limited to wireline network however. As personal communication and ubiquitous access becomes more prevalent, it is necessary to come up with some robust solution which can support multiple applications such as mobile IP-telephony, multimedia and other streaming application over wireless IP network. Supporting multimedia operation over wireless links would have to consider several factors, such as signaling, quality of service, bandwidth management, mobility management and authentication etc.

With a view to realizing different protocols and components needed for supporting mobile streaming multimedia such as wireless IP telephony, broadcasting streaming content in a $3 \mathrm{G}$ wireless IP environment, a testbed has been designed and implemented where the proof of concept for different functional component of a 3G-IP network can be demonstrated.

This testbed illustrates a blue-print of a 3G-IP network [1] that would also involve providing support for roaming across different carrier domains (e.g., micro, macro,and domain mobility), with a provision for billing and network management. It also touches upon the integration with $2 \mathrm{G}$ network by means of interaction with other signaling protocols

(c) 2001 Telcordia Technologies, Inc. such as MGCP [2] and other associated gateways based on SIP [3].

This paper is organized as follows. Section II briefly discusses the testbed architecture and how different requirements for Wireless Internet are realized, section III defines the different components associated with the testbed. Section IV describes the details of of several functional modules implemented. Section V concludes the paper with some open issues.

\section{TESTBED ARChitecture}

This testbed is an embodiment of the components such as signaling, registration, binding, authentication, and QoS which are part and parcel to Mobile Wireless Internet. Proposed testbed emulating a wireless Internet is built upon Linux, Windows and Solaris based platform. Software components used in the testbed are based on the standard IETF protocols such as SIP (Session Initiation Protocol), and SDP (Session Description Protocol) [4] for signaling and media description respectively, RTSP [5] (Real-Time Streaming Protocol) for multimedia streaming, mobile IP or its variant for binding , DHCP [6] or its variant (DRCP) [7] for configuring the client and diffserv based protocols for QoS such as DSNP (Dynamic SLS Negotiation Protocol) [8] as the mobile moves from one subnet to another. IEEE $802.11 \mathrm{~b}$ based wavelan base stations are being used as access point to the wired network. New protocols are developed or extensions are made to the existing ones to provide all the features of a 3G-IP network, such as faster registration, configuration, binding, mobility and QoS. Following subsections describe how different features are implemented.

\section{A. Signaling}

This architecture is built with a vision of 3G-IP network where the end-systems are assumed to be IP end-points, although a possible transition from $2 \mathrm{G}$ to $3 \mathrm{G}$ network has also been tried with interaction of IP and non-IP end points. These IP addresses can be part of the global unique IP address space or can be privately owned in which case addressing can be resolved using NAT (Network Address Translation) or IP masquarading. Because of the distributed nature of this network, Session Initiation Protocol (SIP) has been used 
to demonstrate the signaling and initiation of multi-media call, and its tear down, between the clients. SIP server, SIP user agent are part of the signaling architecture, although SIP server functionality can easily be integrated into the Call Agent (Soft Switch) for demonstrating IP-PSTN call features. An end-to-end signaling architecture has been shown here. While SIP takes care of signaling of multimedia calls between multiple parties, there has been proposal to extend SIP so that it can take care of mobility for mid-session call. HMMP (Host Mobility Management Protocol) a framework [9] based on an extension of SIP has been submitted to IETF for consideration. This method provides an alternative approach to Mobile IP for binding issues and can also take care of pre-session mobility by means of unique URI registration.

\section{B. Dynamic Registration and Configuration}

Registration and configuration involves registering with the network and configuring the end point itself. DHCP, PPP, and Mobile IP are standard ways of providing the registration facility to the end clients. However, Dynamic and Rapid Configuration Protocol is a lightweight version of DHCP that takes care of faster registration and configuration keeping in mind the efficient use of scarce wireless bandwidth. It does so by shrinking the message size, minimizing the messages in transaction and limiting the use of broadcast. As a sister protocol to DHCP, DRCP would have the mechanism to provide the DNS information, lease negotiation and would provide with client server authentication mechanism.

There are many ways subnet discovery can be taken care of: it can be done using detection of layer 2 changes or by server discovery method similar to router discovery mechanism often employed in Mobile IP. This can be achieved by modifying the client code of DHCP so as to trigger either MIP update or SIP re-invite upon detection of a new subnet. Similar changes are built-in features of DRCP, and it can be easily taken care of by server discovery method.

\section{Binding}

Binding allows continuous connectivity of TCP and UDP streams when the communicating end-nodes are moving around. Binding between the mobile host and correspondent host when the mobile host is moving is typically taken care of by Mobile IP [10] or by one of its many variants [12], [13] although it suffers from some drawbacks such as triangular routing and encapsulation. Although there are other alternative solutions such as Cellular IP [11], HAWAII [13], TeleMIP [12] to provide mobility within a domain. MosquitoNet's [14] Mobile IP version without Foreign Agent is used as one of the alternatives in the testbed, to take care of the binding issues. In this case, care of address is typically provided by the DHCP server or DRCP server. While traditional Mobile IP based approach takes care of the binding problems we have used an application layer technique based on SIP based mobility management to take care of personal, and terminal mobility [15]. A framework called Host Mobil-

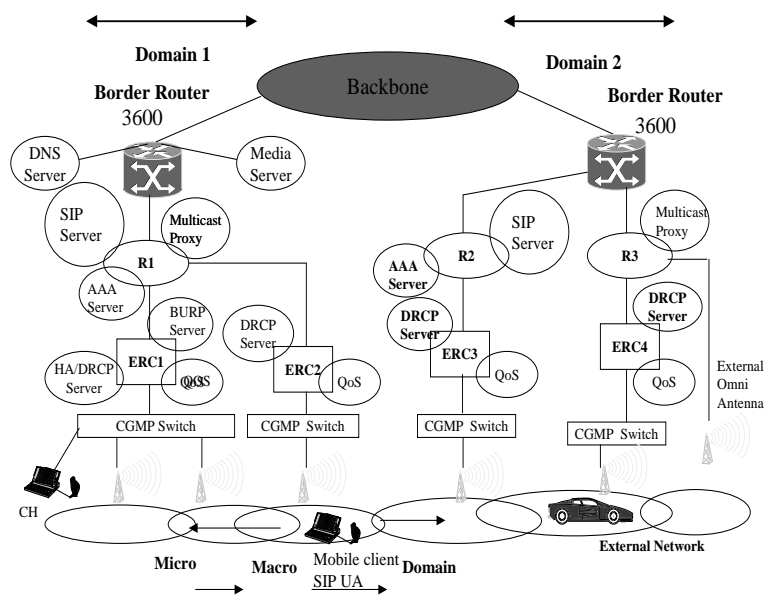

Fig. 1. Basic testbed

ity Management Protocol which is based on SIP's extensions describes this in more details.

It proposes some extension of SIP where by mobility of multimedia calls (RTP/UDP based stream) and TCP application can be taken care of without using underlying Mobile IP and any network components in the middle of the network.

\section{Authentication, Authorization and Accounting}

Current testbed is using AAA protocol DIAMETER for Inter domain mobility, but a user to network version of AAA protocol is used between the local client and the registration agent called Basic User Registration Protocol (BURP). This provides a user level registration to the mobile clients within a domain. Details of this protocol can be found in an Internet draft [16]. BURP can easily integrate with SIP to provide necessary registration mechanism with proper security association.

\section{TESTBED COMPONENTS}

The proposed testbed consists of many hardware components and associated software and protocols. A picture of the current testbed is shown in figure 1 .

\section{A. Mobile Station (Node) (MN)}

Mobile stations are multi-media laptops and PDAs equipped with camera, and audio devices and have PCMCIA slots for wavelan interface to communicate with the base station. Each of these Mobile Stations has multi-media application such as (wb, vic, rat) running in order to establish a multimedia calls.

\section{B. Base Station (BS)}

Base stations are Lucent's IEEE 802.11b based wavelan access points operating at $2.4 \mathrm{GHZ}$ frequency range. Each of these has an IP address which is addresseable through SNMP 
agent. Each base station also operates on a separate 802.11 channel to avoid the possibility of interference. Yagi array antenna are used to extend the lab to outside and provide external field coverage for upto 2 miles.

\section{Base Station Controller (BSC)}

There is currently no hardware Base Station Controller (BSC) which would in practice can control several base stations. Alternatively the base stations are connected to the router directly through stationed network. Base Station Controller is assumed to be a multi-port switch which can control the respective base stations connected to each of its ports. It could be assumed to be a switch capable of controlling the Base Stations based on the MAC address, however these base stations and base station controllers are part of the same subnet. In some cases BSC can be an ether switch capable of filtering multicast packets, and being able to prioritize the traffic destined for a particular mobile host connected to a base station, providing proper QoS in terms of bandwidth.

\section{ERC (Edge Router and Controller)}

ERCs are Linux PCs acting as routers with multiple ethernet interfaces. There are multiple base stations connected to the interface of each router via Cisco's VLAN switches. Each interface of ERC is on a separate subnet. We assume each ERC to be part of the same domain here. In our experiment there are two main routers each dedicated to act as a domain router for each domain.

A separate interface has been dedicated for external network which is tested in the field outside the building.

\section{E. SIP server/SIP User Agent}

SIP user agent runs on all the communicating nodes. Client version of SIP is a tcl/tk and $\mathrm{C}$ code, and provides a nice user interface for managing the multimedia calls. SIP server daemon (sipd) is run on a server, thus making it either a proxy/redirect or registrar. SIP server is not used when the session is established using direct mode. When a call is established using a SIP server (non-direct mode), it can interact with location server, and can be used for services like registration, security and location updates. SIP User agent and servers have also been used for providing personal and terminal mobility support.

\section{F. DRCP server/client}

As explained earlier DRCP is a lightwieght version of DHCP suitable for wireless roaming. There is a DRCP server in each subnet. In some cases when there are multiple interfaces on a server, drcp daemon (drcpd) is started on each interface but with different configuration file. This is responsible for leasing IP addresses to clients, when they request for IP addresses. DRCP client daemon runs in each terminal which gets the IP address from the server upon boot up or after entering to a new subnet. This code has been modified to speed it up by interacting with IEEE 802.11 b driver, Mobile
IP and SIP user agent. As part of the built-in mechanism of DRCP the client would default to DHCP in the absence of DRCP servers.

\section{G. Mobile IP Server/Client}

MosquitoNet's Mobile IP is integrated in the testbed. It can operate without foreign agent in order to provide the care of address, rather DRCP server dispenses it to the client upon a DISCOVER message from the client. Mobile IP server daemon runs in the home agent and client daemon runs in the mobile client. Mobile IP client daemon is started before it makes a move, and it is restarted each time after the mobile node gets a new address on moving to a new subnet. There are many configuration files that need to be maintained in the server and clients to support different modes of operation such as reverse tunneling to take care of the fire wall problems and policy table to be able to decide between real-time and nonreal-time traffic.

\section{H. Media Server}

The application server is a Pentium PC running Linux and run multiple daemons for variety of application such as streaming, web access and IMT2000 emulation. So logically application server consists of a Real Stream server (Media Server), Apache HTTP server, and IMT 2000 emulation server. Practically all these application can co-exist in the same server or stay distributed.

In the absence of a CDMA 2000 based infrastructure, IMT 2000 functionality has been emulated to take advantage of some of the features such as assigning priority of services for a particular kind of application (signaling, data transfer, ftp, http) for a certain host pair, variable speed (e.g., $14.4 \mathrm{kbps}$, $384 \mathrm{kbps}, 2 \mathrm{Mb} / \mathrm{s}$ ), variable state (active/dormant)

This emulation software provides the flexibility to the client to request a certain kind of service (e.g., prioritized bandwidth) for a certain kind of application. For example, signaling traffic may be assigned one particular type of priority than the actual data traffic. Even for data traffic, http may get better priority than the ftp traffic, which can be done based on port pair being used. IMT 2000 emulation software has been developed using tcl/tk and $\mathrm{C}$.

\section{Functional Features}

Some of the current features that can be demonstrated in the testbed are listed below.

1. Multimedia call setup using SIP signaling

2. Seamless mid-Session mobility (micro, macro, and domain) using both Mobile IP and SIP based re-invite mechanism

3. Fast-handoff and registration using light weight version of DHCP (e.g.,DRCP)

4. IMT2000 emulation (Priority of traffic assigned based on application and source-destination pair)

5. QoS features for mobile clients 
6. Authentication, Authorization and Accounting (Interdomain and Intradomain)

7. Wireless Multicast support

8. IP-PSTN integration

Following sections provide methods of operation for some of the features

\section{A. Session Establishment}

Both the correspondent host and mobile host which run Linux operating system obtain their IP addresses from a DRCP server upon boot-up.

Once the clients are up and running, SIP User Agent (sipc) is started up in both the clients. The correspondent host $(\mathrm{CH})$ establishes a call to the mobile client (which may be present in the same subnet) with proper media description by using SDP. This multimedia call can be direct or additionally a SIP server can also be used while the call is being set up, so that security and authentication can be demonstrated. The callee answers the call with its proper media preference. Once both the clients agree on a set of media preferences, the call is established. This call may involve voice, video and data all together. SIP helps to set up the call, but after that actual media flows as RTP/UDP stream between two end-points using the standard routing mechanism. We have used white board (wb) application to demonstrate data sharing, (rat) to demonstrate voice exchange, and (vic) to demonstrate video communication. All these three media streams use different port numbers for each application. IMT 2000 emulation can potentially be used to assign priority to each of these application based on their port number, and also a different priority can be assigned to actual SIP signaling since it uses a separate port number (5060) to be specific. At any point of time either caller or callee can send BYE so that the call terminates. Alternatively this multi-media call can be set up using proxy servers.

\section{B. Mobility of Multimedia stream}

Mobility can be categorized broadly into two kinds: presession mobility and mid-session mobility. Pre-session mobility can be taken care of by proper pre-registration. Current Mobile IP and DHCP/DRCP protocols provide traditional ways to take care of pre-session mobility using layer three technology. However SIP can be used to address presession mobility by means of registration and re-direction using unique URI scheme, an application layer identifier. However mid-session mobility is something which is more important and can be sub-divided into three different categories such as, micro, marco, and domain. Mid-session mobility can be taken care of by using standard Mobile IP approach or application layer approach such as SIP.

DRCP/DHCP daemon runs on the designated servers in the respective subnets. After the SIP multimedia call is established and the RTP media streams flow in duplex mode, mid-session mobility can be demonstrated.

Micro mobility is defined as the mobility when the client moves between two cells, but the base stations involved are part of the same subnets. In this case the client does not change its address however, thus neither mobile IP is triggered nor SIP re-invite is required to be sent to maintain the mobility intact. Handoff is taken care of based on layer two signal strength of the cells. Figure 1 shows as to how micromobility has been demonstrated in the testbed.

Macro mobility is defined when the mobile client moves further away to a different cell, and in this case this new cell is part of a different subnet. It has been shown in figure 1 . As soon as it moves to a new subnet domain, the client discovers (via server advertisement message or sensing a different 802.11 channel number) that it is in a new subnet domain and triggers a DRCP DISCOVER message. The designated DRCP server in the second subnet responds with a new IP address. The mobile client now gets configured with a new IP address. As soon as it obtains the new IP address, mobile IP client daemon is updated on the mobile client and it sends a registration message to the home agent, thus it knows the whereabouts (new IP address) of the mobile host. So the RTP stream destined for the mobile client gets tunneled through the home agent, which gets decapsulated at the mobile client. In case of SIP, using application layer mobility scheme a reinvite is sent to the corresponding host so as to redirect the traffic.

Domain mobility has been demonstrated by extending the mobility to outside of the lab space and configuring a separate DNS domain. There is one host acting as primary DNS server which keeps the database for both the DNS domains. By means of Yagi array antenna, coverage of wavelan testbed has been extended to outside of the building. Dynamic DNS is implemented to provide the update as the IP address changes. Seamless mobility can be obtained even in the case of domain handoff.

Fast handoff is associated with each type of mobility. Fast handoff of multimedia stream is typically dependent upon quick detection, faster reconfiguration, and registration with the new subnet/domain and re-direction of the stream after detection of the new subnet. Standard version of DHCP takes about 10-15 sec without ARP checking [17], it offers $5 \mathrm{sec}$ latency (with ARP checking suppressed), and it is about 100 msec with DRCP. DRCP offers the lowest time for latency because of the factors mentioned in the earlier section.

\section{Wireless IP Multicast}

In order to achieve better bandwidth efficiency and make the group communication (many-to-many) and mass communication (e.g., audio/video streaming from a single source) more effective, IP Multicast has been deployed in this testbed. There are many issues related to multicast mobility (e.g., continue to be part of the same group while changing cells, subnets etc.), use of a smart base station controller to restrict the multicast stream to one cell after the listener moved, are being looked into by using IGMP snooping or CGMP.

Currently DVMRP has been installed on the Linux based routers in the test-bed, Cisco routers are running PIM, there is 


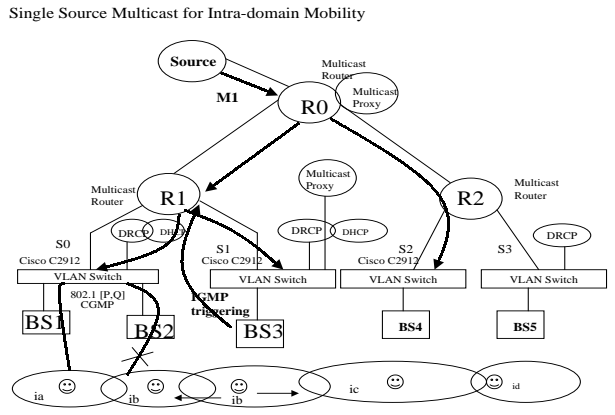

Fig. 2. Multicast flow

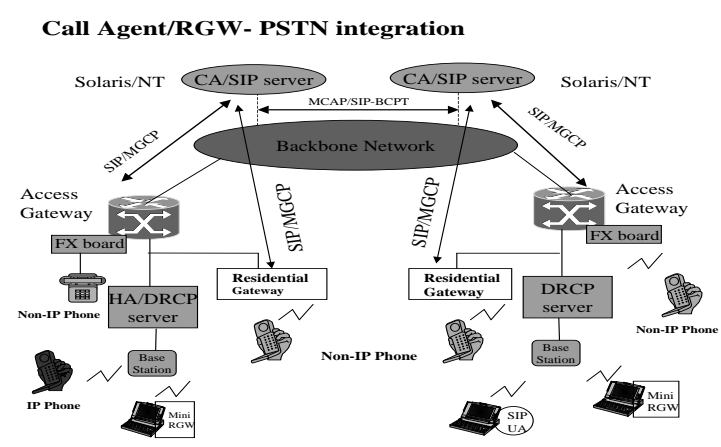

Fig. 3. Call Agent Realization
PIM-DVMRP tunneling between Cisco routers and the Linux based routers running mrouted [18] so as to enable the multicast support. SIP signaling can also be used to take advantage of the IP multicast to invite a group of people to a conference, invite a stream server to a conference, move from a two party conference to a multi-party conference and help providing virtual soft-handoff. This multicast capability can also be used to take care of mobility for single streaming source.

A standard multicast testbed configuration has been shown in figure 2 .

\section{Wireless Call Agent/SIP-PSTN integration}

In addition to providing mobile multimedia support between the IP end-points, this test-bed also provides a way of integrating the PSTN components by using Call-Agent (Media Gateway Controller) and SIP server. Call Agent is based on Media Gateway Control Protocol, where Media Gateway Controller is resident on a server, and would control the nonIP devices connected to the gateway. This gateway is usually a media gateway, whose interface is connected to the IP cloud. The other interface usually connects to a standard analog phone, or a PBX. Media gateway converts the analog signal to IP stream, and has a MGCP slave agent which is controlled by the Media Gateway Controller. In this testbed the gateway is nothing but a Cisco router with an FX board which connects to a standard analog phone. The call agent maintains a database of the PSTN end-points, and would have access to intelligent database which would provide some AIN (Advanced Intelligent Networking) functionality. In order to provide scalability or domain connectivity there can be multiple call-agents. The protocol between the call-agents can be SIP-T [25], an open standard protocol.

A standard Call-Agent configuration has been shown in figure 3. There is an altrenative way of using MGCP while not using the standard residential gateway. In this case a mini residential gateway has been implemented as a software on the mobile terminal itself and the audio stream can be generated from the terminal itself by using a terminal emulator. This way MGCP can be used on a mobile terminal. Mobility in this case has been tried using standard Mobile IP in windows environment although application layer mobility is not ruled out.

SIP-PSTN integration has been realized by using a pair of mediatrix gateway [26], analog cordless phones and windows based SIP server which keeps a mapping between the end points. In this scenario each mediatrix box has SIP User Agent in it, which genarates and terminates the SIP signal.

\section{CONCLUSIONS AND OPEN IsSUES}

This paper described different techniques associated with building a wireless Internet testbed. It touches upon the realization steps for real-life Internet roaming, by providing the implementation details of several components associated with the testbed. These include all IP wireless multimedia calls over a mobile environment, authorization, QoS, multicast and IP-PSTN interaction. Some of the future work would involve providing flexible streaming services such as SIP based voice mail service, location based mobile services using mobile Application Server and heterogeneous access.

\section{ACKNOWLEDGEMENT}

Authors would like to acknowledge the effort of other ITSUMO team members and Parmesh Ramanathan of University of Wisconsin towards their contribution for realization of different components of the testbed. Help from Xiaotao $\mathrm{Wu}$ and Jonathan Lennox of Columbia University towards sipc and sipd are also appreciated.

\section{REFERENCES}

[1] www.mwif.org

[2] M. Arango et al., "Media Gateway Control Protocol", RFC 2705, Internet Engineering Task Force

[3] M. Handley, H. Schulzrinne, E. Schooler, and J. Rosenberg, "SIP: Session initiation protocol," RFC 2543, IETF, March 1999.

[4] M. Handley and V. Jacobson, "SDP: session description protocol," Request for Comments (Proposed Standard) 2327, Internet Engineering Task Force, Apr. 1998.

[5] H. Schulzrinne, A. Rao, and R. Lanphier, "Real time streaming protocol (RTSP)," Request for Comments (Proposed Standard) 2326, Internet Engineering Task Force, Apr. 1998.

[6] R. Droms, "Dynamic host configuration protocol," RFC 2131, IETF, March 1997.

[7] A. Mcauley, S. Das et al, "Dynamic Rapid Configuration Protocol", Internet Draft, work in progress

[8] J.C Chen, A. Caro, A MCauley, "A QoS Architecture for Future Wireless IP Networks" 2000, submitted for QoS workshop, Las Vegas 
[9] F. Vakil, A.Dutta, J.C Chen, S. Baba et al, "Host Mobility Management Protocol", Internet Draft, work in progress, December 2000

[10] C.E. Perkins, 'IP mobility support," RFC 2002, IETF, October 1996.

[11] A.T. Campbell, J. Gomez, S. Kim, A.G. Valko, C-Y. Wan and Z. Turanyi, "Design, implementation, and evaluation of Cellular IP," IEEE Personal Commun. Mag., vol. 7, no. 4, August 2000.

[12] S. Das, A. Misra, P. Agrawal, S.K Das, "TeleMIP: Telecommunications-enhanced Mobile IP architecture for fast intradomain mobility," IEEE Personal Commun. Mag., vol. 7, no. 4, August 2000.

[13] R. Ramjee, T.L. Porta, S. Thuel, K. Varadhan, and S.Y. Wang, "HAWAII: A domain-based approach for supporting mobility in widearea wireless networks," in IEEE Intl. Conf. on Network Protocols (ICNP'99), Toronto, Canada, November 1999.

[14] mosquitonet.stanford.edu

[15] E. Wedlund and H. Schulzrinne, "Mobility support using SIP," in ACM WoWMoM'99, Seattle, Washington, August 1999.

[16] S. Das, A. Mcauley et al, "Basic User Registration Protocol", Internet Draft, work in progress, BURP BOF 50th IETF.

[17] Jon-Olov Vatn, Gerald Maguire, "The effect of using co-located care-of addresses on macro handover latency"

[18] http://www.jukie.net/bart/multicast/Linux-MroutedMiniHOWTO.html

[19] A. Dutta, F. Vakil, J-C. Chen, M. Tauil, S. Baba, N. Nakajima, and H. Schulzrinne, ”Application Layer Mobility Management Scheme for Wireless Internet," in IEEE 3GWireless'01, San Francisco, CA, May 2001.

[20] Satwant Kaur et al, "Multicast Support for mobile IP using a modified IGMP" in the Proceedings of the IEEE Wireless Communications and Networking Conference, 1999 (IEEE WCNC'99), September 1999.

[21] A.J. McAuley et al, "Mobile Multicast Proxy", ATIRP conference

[22] Beau Williamson,"Developing IP Multicast Networks".

[23] H. Schulzrinne, S. Casner, R. Frederick, and V. Jacobson, "RTP: a transport protocol for real-time applications," Request for Comments (Proposed Standard) 1889, Internet Engineering Task Force, Jan. 1996.

[24] "UCL RAT http://www-mice.cs.ucl.ac.uk/multimedia/software/rat"

[25] Aparna Vemuri, Jon Peterson "draft-vemuri-sip-t-context-02.txt" Internet Engineering Task Force

[26] http://www.mediatrix.com 